\title{
Canonical Representation of the Active Nanoparticles Kinetics
}

\author{
Olga V. Yushchenko*, Anna Yu. Badalyan \\ Nanoelectronics Department, Sumy State University, 2 Rimskii-Korsakov Str., Sumy, 40007, Ukraine \\ *Corresponding Author: yushchenko@phe.sumdu.edu.ua
}

Copyright (C2014 Horizon Research Publishing All rights reserved.

\begin{abstract}
Within the framework of the canonical Hamilton system the behavior of active nanoparticles was investigated. On the basis of the phase portraits the kinetics of the system was studied. The transformations of the nanoparticle's internal energy into the kinetic energy of the motion and into the total mechanic energy were considered.
\end{abstract}

Keywords Active Nanoparticles,Hamilton Function, Internal Energy

\section{Introduction}

Starting with the famous lecture "There's Plenty of Room at the Bottom" by Richard Feynman the interest in nanoscale systems and their applications is still increasing. Besides the development of new materials is closely linked with the progress in nanoscience. In this case there are two basic technologies: top-down and bottom-up [1]. The latter is a more complicated technique, because it involves the process of self-organization (or self-assembly) of individual nanoparticles. Thus the control of the nanoparticles' motion is on the top of interest today.

In recent years, a lot of experiments are conducted with so-called Janus nanoparticles - the gold-capped colloidal spheres, which are heated by laser light and as a result can perform various types of the motion $[2,3,4$, $5,6]$. This happens because the particles transform their internal energy into the energy of motion $[4,7,8,9,10]$. Such behavior of gold nanoparticles is very similar to the motion of biological objects (bacteria, cells, macromolecules, plankton, etc.), which also have a store of the internal energy $[11,12,13,14,15,16,17,18,19,20]$. Therefore the motion of active nanoparticles ${ }^{1}$ are sometimes identified with self-proppelled motion in biology $[4,10,18,22,23]$. Although it is worth noting that the active particles (or self-phoretic colloids) and so-called run-and-tumble particles (motile bacteria) are not always equivalent: the paper [24] (where the criterion of equivalence are obtained) discusses the relationship of these two concepts.

In many studies $[5,6,7,9,16,17,18,19,25]$ the motion of biological objects and nanoparticles reduced to three main types (see Fig.1): translational, rotational and run-and-tumble motion.

In our consideration we propose an analytical model, which allows to describe the motion of nanoparticles in a self-consistent way. Despite the many different analytical and numerical methods $[3,10,26,27,28$, $29,30,31,32,33]$ for describing such behavior a consistent analytical scheme based on the canonical representation does not exist yet. Besides canonical approach we take into account the similarity with the motions of biological objects and develop previous work $[5,6,7,10,27,30,31,32]$ in this direction.

\section{Basic equations}

We can describe the kinetics of the dynamical system using the phase space $(\mathbf{q}, \mathbf{p})$, where $\mathbf{q}=q_{1}, q_{2}, \ldots, q_{n}$ is the $n$-dimensional coordinate of the particle and $\mathbf{p}=$ $p_{1}, p_{2}, \ldots, p_{n}$ is its $n$-dimensional momentum $[34,35]$. The point on the phase space represents a state of the system. Changes in the state of the system lead to a

\footnotetext{
${ }^{1}$ The term "active Brownian particle" was first proposed in 1995 in Ref.[21] for the particles, which can generate the field that defines the particles' motion.
} 

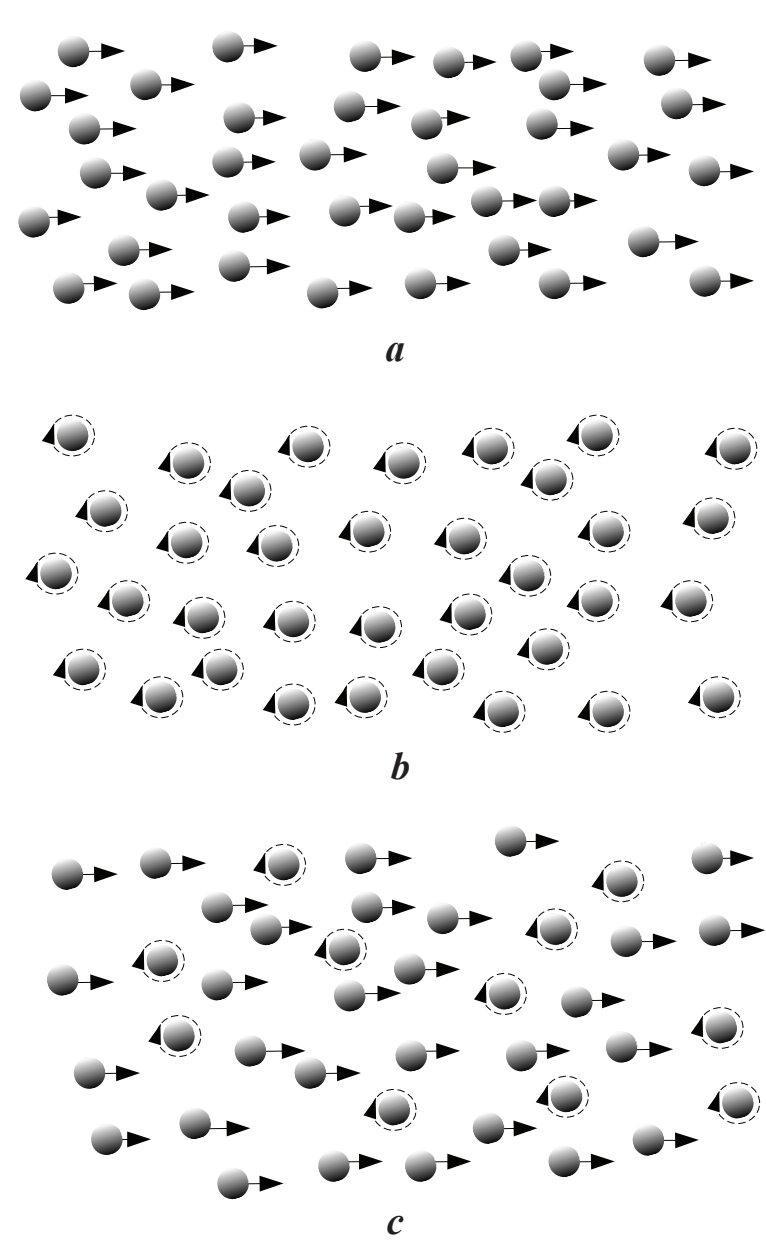

Figure 1. Schematic representation of the types of nanoparticles motion a) translational motion, b) rotational motion, c) run-andtumble motion

displacement of the point in the phase space - as a result we obtain the phase trajectory. So, having a phase portrait (a set of phase trajectories) of the system we can describe the behavior of nanoparticle ensemble.

The phase flow $\hat{F}$ describing the changes in the system state

$$
(\mathbf{q}(t), \mathbf{p}(t))=\hat{F}(\mathbf{q}(0), \mathbf{p}(0))
$$

is defined by the differential equations of the motion $[34,35]$

$$
\begin{aligned}
\dot{\mathbf{q}} & =Q(\mathbf{q}, \mathbf{p}, t), \\
\dot{\mathbf{p}} & =P(\mathbf{q}, \mathbf{p}, t)
\end{aligned}
$$

(where dot denotes differentiation with respect to time $t)$.

For determining the specific form of these equations we must apply the corresponding conservation law the conservation of the phase volume - which defines a class of Hamilton systems with motion equations

$$
\begin{aligned}
\dot{\mathbf{q}} & =\frac{\partial \mathcal{H}}{\partial \mathbf{p}}, \\
\dot{\mathbf{p}} & =-\frac{\partial \mathcal{H}}{\partial \mathbf{q}} .
\end{aligned}
$$

Here the Hamilton function

$$
\mathcal{H}(\mathbf{q}, \mathbf{p}, t)=\mathcal{K}(\mathbf{p}, t)+\mathcal{P}(\mathbf{q}, t)
$$

represents the total mechanical energy of the system, i.e. the sum of kinetic $\mathcal{K}$ and potential $\mathcal{P}$ energy.

In $[30,31]$ the authors proposed a general theory of canonical dissipative systems with couplings of the internal energy. Following this idea we consider the nanoparticles motion in a medium with liquid friction. For this purpose in the equation (3) we add the term $\dot{\mathbf{p}} \propto-\gamma \mathbf{p}$, which represents the friction force of a solid sphere in the liquid/gas medium at low Reynolds numbers ( $\gamma$ is a liquid friction coefficient) [10, 23].

Describing the active motion we must also include in our analysis the internal energy $\mathcal{E}$ of the particle and its possible transformations. As a result on the basis of the canonical system (2)-(3) we get three self-consistent differential equations

$$
\begin{aligned}
\dot{\mathbf{q}} & =\frac{\partial \mathcal{H}}{\partial \mathbf{p}} \\
\dot{\mathbf{p}} & =-\frac{\partial \mathcal{H}}{\partial \mathbf{q}}-\gamma \frac{\partial \mathcal{H}}{\partial \mathbf{p}}+\beta \mathcal{E} \cdot f_{1}(\mathcal{H}), \\
\dot{\mathcal{E}} & =\frac{\mathcal{E}_{e}-\mathcal{E}}{\tau}-2 \mu \mathcal{E} \cdot f_{2}(\mathcal{H}) .
\end{aligned}
$$

Equation (7) for the internal energy contains relaxation and dissipation terms: the first term takes into account the decrease in the internal energy in stand-alone mode to the value $\mathcal{E}_{e}$ defined by external conditions ( $\tau$ is the corresponding relaxation time); the second (dissipation) term describes the decrease in the internal energy after its transformation into kinetic or total mechanical energy. At the same time this transformation introduces an additional positive term in the equation (6). The form of the functions $f_{1}$ and $f_{2}$ depends on the type of energy transformation ( $\beta$ and $2 \mu$ are the positive coupling constants).

A description of the collective motion of active particles is often reduced to the one-body problem [10, 27]. So we simplify our system to the one-dimensional case using the notations $\mathbf{q} \rightarrow q, \mathbf{p} \rightarrow p, \mathcal{H} \rightarrow H, \mathcal{K} \rightarrow K$, $\mathcal{P} \rightarrow P, \mathcal{E} \rightarrow \varepsilon$. Then, instead of rotational motion, we can consider the oscillations along a single axis. The one-dimensional case is the most simple, but on its example the important concepts of the general theory can be studied. Furthermore for an ensemble of identical 
particles we can use a set of different initial conditions (coordinates and momenta) for single nanoparticle when solving the system (5)-(7).

\section{Transformation of the internal energy into kinetic energy}

The most common form of the Hamilton function

$$
H=K(p)+P(q)=\frac{p^{2}}{2 m}+\frac{m \omega^{2} q^{2}}{2},
$$

is determined by a particle mass $m$ and natural frequency $\omega[36]$.

First, we consider the transformation of the internal energy only into the kinetic energy. In this instance the system (5)-(7) becomes

$$
\begin{aligned}
\dot{q} & =\frac{\partial H}{\partial p}, \\
\dot{p} & =-\frac{\partial H}{\partial q}-\gamma \frac{\partial H}{\partial p}+\beta \varepsilon \frac{\partial H}{\partial p}, \\
\dot{\varepsilon} & =\frac{\varepsilon_{e}-\varepsilon}{\tau}-2 \mu \varepsilon K(p) .
\end{aligned}
$$

Substituting the Hamilton function (8) into Eqs.(9)(11), we obtain

$$
\begin{aligned}
\dot{q} & =\frac{p}{m}, \\
\dot{p} & =-m \omega^{2} q-\gamma \frac{p}{m}+\beta \varepsilon \frac{p}{m}, \\
\dot{\varepsilon} & =\frac{\varepsilon_{e}-\varepsilon}{\tau}-\mu \varepsilon \frac{p^{2}}{m} .
\end{aligned}
$$

This system of equations has a lot of constants, making it difficult to study. Therefore, we introduce the dimensionless variables (time, coordinate, momentum and internal energy) using the following scales in terms of dimension

$$
t_{s} \equiv \frac{m}{\gamma}, \quad q_{s} \equiv\left(\frac{m}{\mu \tau \gamma^{2}}\right)^{\frac{1}{2}}, \quad p_{s} \equiv\left(\frac{m}{\mu \tau}\right)^{\frac{1}{2}}, \quad \varepsilon_{s} \equiv \frac{\gamma}{\beta} .
$$

Then in dimensionless form the system (12)-(14) reduces to

$$
\begin{aligned}
\dot{q} & =p \\
\dot{p} & =-\chi q-p+\varepsilon p \\
\delta \dot{\varepsilon} & =\varepsilon_{e}-\varepsilon-\varepsilon p^{2},
\end{aligned}
$$

where we also introduce the dimensionless constants

$$
\chi \equiv t_{s}^{2} \omega^{2}, \quad \delta \equiv \tau / t_{s} .
$$

Since we assume that the internal energy of the system changes quickly, we can apply the approximation

$$
\tau \ll t_{s},
$$

which means that the relaxation time of the internal energy is much less than the time scale. Then $\delta \ll 1$ and the left-hand side of the Eq.(18) can be neglected. As a result we obtain the following dependence of the internal energy on the momentum

$$
\varepsilon=\frac{\varepsilon_{e}}{1+p^{2}}
$$

The Fig. 2 shows that in the stationary mode the in-

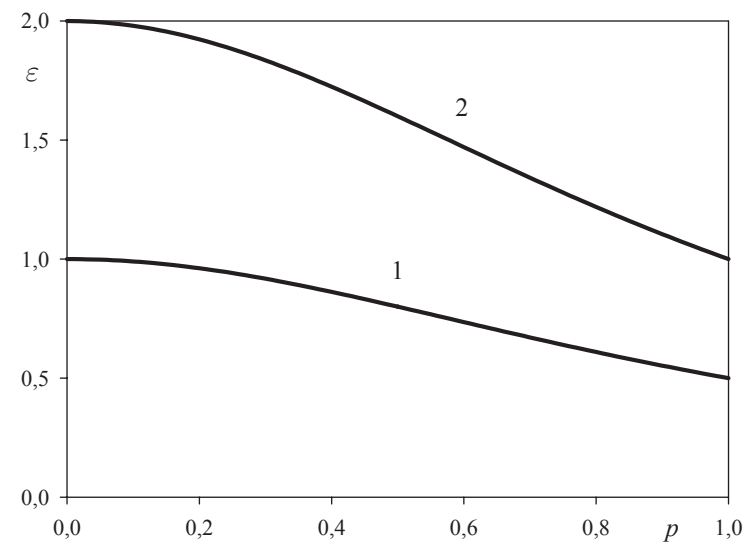

Figure 2. The dependence (21) of the internal energy on the momentum for $\mathbf{1}-\varepsilon_{e}=1 ; \mathbf{2}-\varepsilon_{e}=2$

ternal energy decreases from the value $\varepsilon_{e}$, given by the external conditions, to a value $\varepsilon_{e} / 2$ at the maximum momentum $p_{m}=\sqrt{m /(\mu \tau)}$.

Substituting the expression (21) into the Eq.(17) we obtain the system of two differential equations

$$
\begin{aligned}
\dot{q} & =p, \\
\dot{p} & =-\chi q-p+\frac{\varepsilon_{e} p}{1+p^{2}},
\end{aligned}
$$

instead of (16)-(18). Now we can build the phase portrait and analyze the kinetics of the system in the phase space $(q, p)$.

First we define the singular points corresponding to the equilibrium states of the system. For Eqs.(22) there is only one singular point $O$ with the coordinates $q_{0}=0$, $p_{0}=0$.

The behavior of the phase trajectories near the singular point $O$ can be analyzed by Lyapunov exponents $\lambda$ (for regular mode $\lambda \leq 0$ while in chaotic regime $\lambda>0$ ) 
[34]. When searching the Lyapunov exponents, we apply the substitution

$$
\begin{aligned}
& q=q_{0}+a e^{\lambda t} \\
& p=p_{0}+b e^{\lambda t}
\end{aligned}
$$

where $a, b \ll 1$ are the amplitudes of the deviation from the singular point. As a result from Eqs.(22) we get

$$
\lambda_{1,2}=\frac{\varepsilon_{e}-1}{2} \pm \sqrt{\left(\frac{\varepsilon_{e}-1}{2}\right)^{2}-\chi}
$$

For demonstrating the corresponding variants of phase portraits we combined them with the phase diagram (see Fig.3).

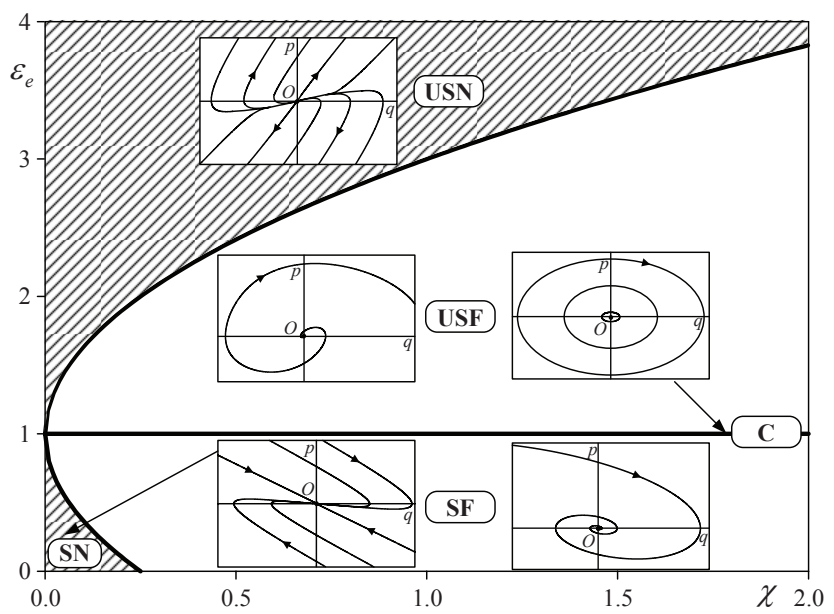

Figure 3. Phase diagram of the system: USN - region of the unstable node realization, the corresponding phase portrait is built for $\chi=1, \varepsilon_{e}=4$; USF - the unstable focus region, phase portrait for $\chi=1, \varepsilon_{e}=2 ; \mathrm{SN}$ - the stable node region, $\chi=0.1, \varepsilon_{e}=0.1$ $\mathrm{SF}$ - the stable focus region, $\chi=1, \varepsilon_{e}=0.5$. Center (C) is realized on the line $\varepsilon_{e}=1$, portrait corresponds to $\chi=1$

Analyzing the Lyapunov exponents (24) we can say that singular point $O$ is stable for $\varepsilon_{e}<1$, and unstable in the opposite case $\varepsilon_{e}>1$. For critical case $\varepsilon_{e}=1$ the point $O$ becomes an elliptical one $(C$ in Fig.3, point $O$ represent a center ${ }^{2}$ ). The damped oscillatory regime, under which point $O$ corresponds to a focus, is realized for $\varepsilon_{e} \in(1-2 \sqrt{\chi} ; 1+2 \sqrt{\chi})$.

According to the phase portraits we can study the kinetics of active nanoparticles. For example, in stable node region (SN in Fig.3, point $O$ represents a stable node) we get a uniform change of the coordinate and momentum that corresponds to the translational type of nanoparticles' motion. For the stable focus region

\footnotetext{
${ }^{2}$ The motion near the center will be stable: the trajectories passing in a small neighborhood of this point will always perform a finite motion.
}

(SF in Fig.3, $O$ is a stable focus) we have damped oscillatory regime when the particle periodically changes its coordinate and momentum with decreasing amplitude. In both these cases, the particles with different initial coordinates and momenta will be assembled at the point $O$ (but in the second case, self-assembly process will be slower).

For unstable regions (USN and USF in Fig.3) the process will be the opposite: for any initial values of the coordinate and momentum the particle will leave the neighborhood of the singular point $O$. And the selfassembly of nanoparticles does not occur.

Thus changing the parameters of the environment (or changing the value of the internal energy $\varepsilon_{e}$ ), we can control not only the type of the nanoparticles' motion but also the assembly process.

\section{Transformation of the internal energy into the total mechanic energy}

If the internal energy is transformed into the total mechanical energy, instead of (9)-(11), we should write

$$
\begin{aligned}
\dot{q} & =\frac{\partial H}{\partial p}, \\
\dot{p} & =-\frac{\partial H}{\partial q}-\gamma \frac{\partial H}{\partial p}+\beta \varepsilon \frac{\partial H}{\partial p}+\alpha \varepsilon \frac{\partial H}{\partial q}, \\
\dot{\varepsilon} & =\frac{\varepsilon_{e}-\varepsilon}{\tau}-2 \mu \varepsilon K(p)-2 \sigma \varepsilon P(q) .
\end{aligned}
$$

Here $\alpha$ and $\sigma$ are the corresponding positive constants of the couplings to the potential energy. Substituting in the system (25)-(27) the Hamilton function (8) we arrive at

$$
\begin{aligned}
\dot{q} & =\frac{p}{m}, \\
\dot{p} & =-m \omega^{2} q-\gamma \frac{p}{m}+\beta \varepsilon \frac{p}{m}+\alpha \varepsilon m \omega^{2} q, \\
\dot{\varepsilon} & =\frac{\varepsilon_{e}-\varepsilon}{\tau}-\mu \varepsilon \frac{p^{2}}{m}-\sigma \varepsilon m \omega^{2} q^{2} .
\end{aligned}
$$

As in the previous case, we use the dimensionless variables with scales (15) and dimensionless constants (19). In addition, we introduce the notations

$$
\varphi \equiv \frac{\alpha \gamma}{\beta}, \quad \psi \equiv \frac{\sigma}{\mu}
$$

Then the system (28)-(30) takes the final form

$$
\begin{aligned}
\dot{q} & =p, \\
\dot{p} & =-\chi q-p+\varepsilon p+\varphi \chi \varepsilon q, \\
\delta \dot{\varepsilon} & =\varepsilon_{e}-\varepsilon-\varepsilon p^{2}-\psi \chi \varepsilon q^{2} .
\end{aligned}
$$


The approximation (20) leads to the dependence of the internal energy (see Fig.4)

$$
\varepsilon=\frac{\varepsilon_{e}}{1+p^{2}+\psi \chi q^{2}}
$$

different from the (21).

As a result the behavior of the system is described by two differential equations

$$
\begin{aligned}
\dot{q} & =p, \\
\dot{p} & =-\chi q-p+\varepsilon_{e} \frac{p+\varphi \chi q}{1+p^{2}+\psi \chi q^{2}},
\end{aligned}
$$

which are characterized by three singular points

$$
O(0 ; 0), C\left(-\sqrt{\frac{\varepsilon_{e} \varphi-1}{\psi \chi}} ; 0\right), D\left(\sqrt{\frac{\varepsilon_{e} \varphi-1}{\psi \chi}} ; 0\right) .
$$

The last two points are realized under the condition $\varepsilon_{e}>\varphi^{-1}$.

Analyzing the stability type of the point $O$, we obtain the following Lyapunov exponents

$$
\lambda_{1,2}=\frac{\varepsilon_{e}-1}{2} \pm \sqrt{\left(\frac{\varepsilon_{e}-1}{2}\right)^{2}-\chi\left(1-\varepsilon_{e} \varphi\right) .}
$$

If the parameter $\varepsilon_{e} \in\left(\zeta_{1}, \zeta_{2}\right)$ where $\zeta_{1,2}=$ $\left(1-2 \chi \varphi \mp 2 \sqrt{\chi(1-\varphi)+\chi^{2} \varphi^{2}}\right)$, the damped oscillatory regime is realized (the singular point $O$ is a focus). At the same time this point will be stable if $\varepsilon_{e}<1$.

Lyapunov exponents for the singular points $C$ and $D$ are given by the equation

$$
\lambda_{1,2}=\frac{1-\varphi}{2 \varphi} \pm \sqrt{\left(\frac{1-\varphi}{2 \varphi}\right)^{2}-2 \chi \frac{\varepsilon_{e} \varphi-1}{\varepsilon_{e} \varphi}} .
$$

For these points the damped oscillatory regime is realized if

$$
\varepsilon_{e}<\frac{8 \varphi \chi}{8 \varphi^{2} \chi-(1-\varphi)^{2}}
$$

Besides points $C$ and $D$ become stable for $\varphi>1$.

We present in Fig.5 some examples of phase portraits of the system (36)-(37). For simplicity, we choose $\psi=1$, since Lyapunov exponents (38),(39) do not depend on this parameter.

In this case, the number of the stable states of the system increases. For example, phase portrait in Fig. $5 c$ represents two stable focuses $C$ and $D$ that may indicate a parallel process of self-assembly. Besides now we can control the coordinate of the self-assembly process: point $O$ for the case shown in Fig. $5 a$ or points $C$ and $D$ in Fig. $5 c$ ).
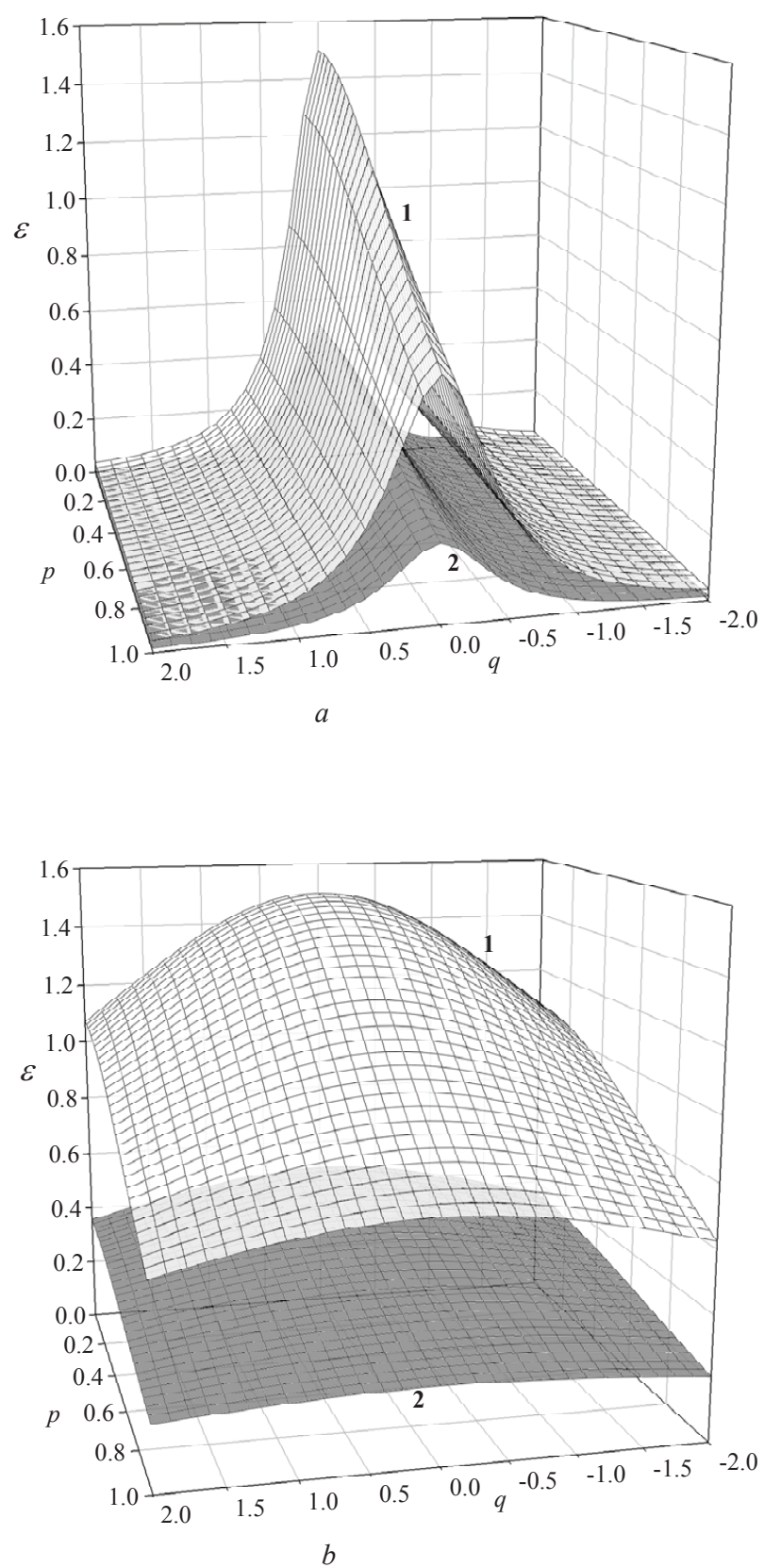

Figure 4. Dependence of the internal energy for $\chi=1$ at $a$ ) $\psi=10, b) \psi=0.1$. Surface 1 corresponds to the $\varepsilon_{e}=0.5$, surface 2 to the $\varepsilon_{e}=1.5$ 


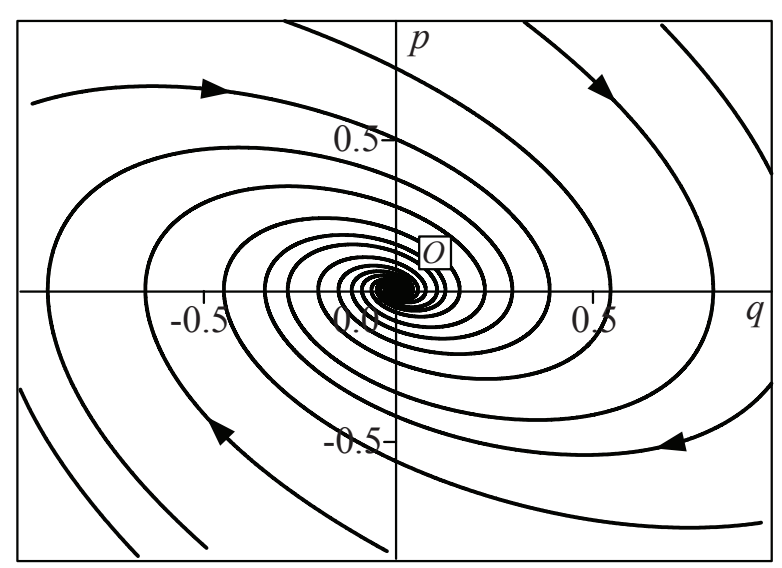

$a$

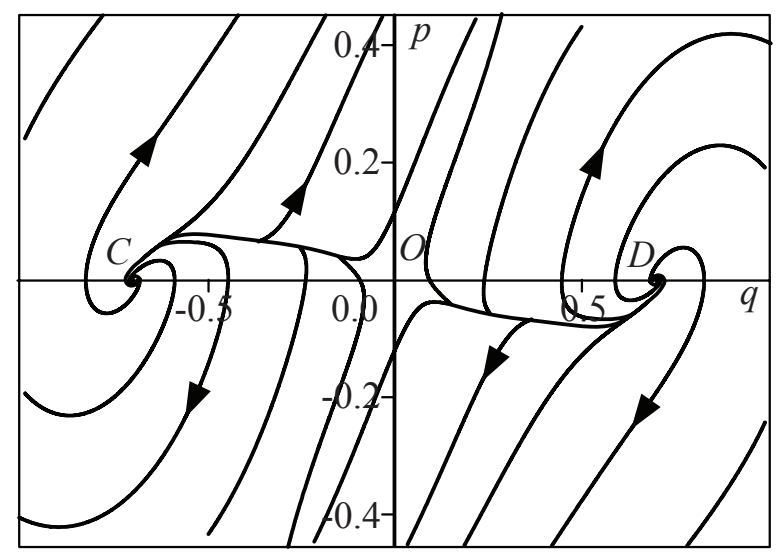

$b$

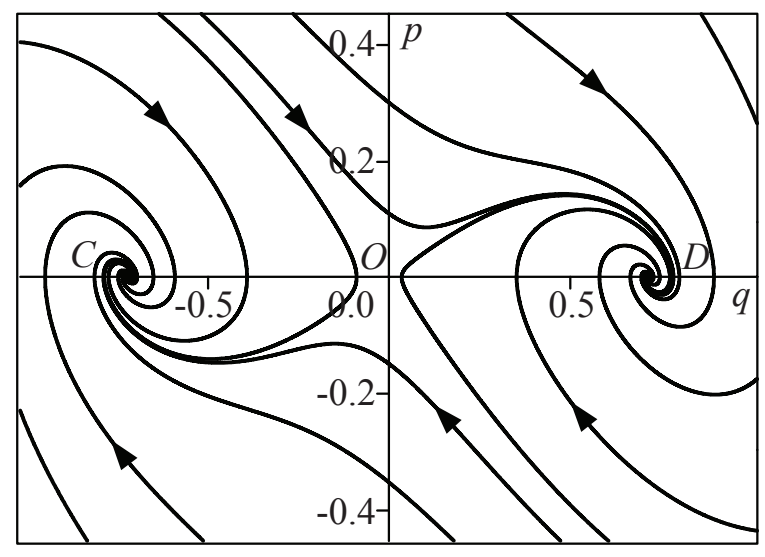

c

Figure 5. Phase portraits of the system (36)-(37) at $\chi=1$, $\left.\left.\psi=1, a) \varepsilon_{e}=0.5, \varphi=0.5 ; b\right) \varepsilon_{e}=3, \varphi=0.5 ; c\right) \varepsilon_{e}=0.5$, $\varphi=3$.

\section{Conclusion}

The control of the nanoparticles' motion are quite actual problem at the moment for many studies.

In our work, we suggested a self-consistent model that allowed to describe the motion of active nanoparticles in the canonical representation. We postulated the equation for the internal energy and introduced the corresponding terms in the Hamilton canonical system of equations. As a result, we investigated the singular points, which corresponded to equilibrium states of the system, and analyzed the kinetics of the system on the example of the phase portraits.

Our findings may help in modeling the processes occurring in an ensemble of identical nanoparticles. Especially it is actual in terms of developing and producing new nanomaterials by bottom-up technique, when you can control the coordinate and the velocity of the process of self-assembly of nanoparticles.

\section{REFERENCES}

[1] A. Biswas, I.S. Bayer, A.S. Biris, T. Wang, E. Dervishi, F. Faupel. Advances in topdown and bottomup surface nanofabrication: Techniques, applications and future prospects, Advances in Colloid and Interface Science 170, 2 (2012).

[2] D. Rings, R. Schacho, M. Selmke, F. Cichos, K. Kroy. Hot Brownian Motion, Phys. Rev. Lett. 105, 090604 (2010).

[3] B. Hagen, S. Teeffelen, H. Löwen. Brownian motion of a self-propelled particle, J. Phys.: Condens.Matter 23, 194119 (2011).

[4] I. Buttinoni, G. Volpe, F. Kümmel, G. Volpe, C. Bechinger. Active Brownian Motion Tunable by Light, J. Phys.: Condens.Matter 24, 284129 (2012).

[5] O. V. Yushchenko, A. Yu. Badalyan. Statiatical description of the collective motion of nanoparticles, Phys. Rev. E 85, 051127 (2012).

[6] O. V. Yushchenko, A. Yu. Badalyan. Study of modes of movement of nanoparticles in the stochastic system, J. Nano- Electron. Phys. 4, 03009 (2012).

[7] F. Schweitzer Active Brownian Particles with Internal Energy Depot in: Traffic and Granular Flow '99: Social, Traffic, and Granular Dynamics Eds. D. Helbing, H. J. Herrmann, M. Schreckenberg, D. E. Wolf (Springer, Berlin 2000) pp. 161-172.

[8] F. Schweitzer, B. Tilch, W. Ebeling. Active Brownian particles with energy depots modeling animal mobility, Eur. Phys. J. B 14, 157 (2000). 
[9] W. Ebeling, F. Schweitzer. Self-organization, active Brownain dynamics and biological applications, Nova Acta Leopoldina NF 88, 169 (2003).

[10] P. Romanczuk, M. Bar, W. Ebeling, B. Linder, L. Schimansky - Geier. Active Brownian Particles. From Individual to Collective Stochastic Dynamics, Eur. Phys. J. ST 202, 1 (2012).

[11] J. Adler. Chemotaxis in Bacteria, Science 153, 708 (1966).

[12] M. Holz, S. Chen. Spatiotemporal structure of migrating chemotactic band of Escherichia coli. I. Traveling band profile, Biophys. J. 26, 243 (1979).

[13] P. Wang, S. Chen. Quasi-elastic light scattering from migrating chemotactic bands of Escherichia coli. III. Studies of band formation propagation and motility in oxygen and serine substrates, Biophys. J. 49, 1205 (1986).

[14] B. Kerner, V. Krinskii, V. Osipov. Thermodynamics and Pattern Formation in Biology, (de Gruyter, Berlin, 1988).

[15] W. Alt, G. Hoffmann Biological motion, (Springer, Berlin, 1990).

[16] G. Ivanitskii, A. Medvinskii, M. Tsyganov, V. Shakhbazian, I. Kreteva. Formation of stationary demarcation zones between population autowaves propagating towards each other, Physica D 64, 267 (1993).

[17] G. Ivanitskii, A. Medvinskii, M. Tsyganov. From the dynamics of population autowaves generated by living cells to neuroinformatics, Physics Uspekhi 37, 961 (1994).

[18] J. Tailleur, M. Cates. Statistical Mechanics of Interacting Run-and-Tumble Bacteria, Phys.Rev.Lett. 100, 218103 (2008).

[19] H.P. Zhang, A. Be'er, E.-L. Florin, H.L. Swinney. Collective motion and density fluctuations in bacterial colonies, PNAS 107, 13626 (2010).

[20] R. Vogel, H. Stark. Force-extension curves of bacterial flagella, Eur. Phys. J. E 33, 259 (2010).

[21] L. Schimansky-Geier, M. Mieth, H. Rose, H. Malchow. Structure formation by active Brownian particles, Phys. Lett. A 207, 140 (1995).
[22] B. Lindner, E.M. Nicola. Diffusion in different models of active Brownian motion, Eur. Phys. J. ST 157, 43 (2008).

[23] V. Lobaskin, D. Lobaskin, I.M. Kulic. Brownian dynamics of a microswimmer, Eur. Phys. J. ST 157, 149 (2008).

[24] M.E. Cates, J. Tailleur. When are active Brownian particles and run-and-tumble particles equivalent? Consequences for motility-induced phase separation, EPL 101, 20010 (2013).

[25] O.V. Yushchenko, A.Sh. Baranova. Self-organization in the collective behavior of active nanoparticles, J. NanoElectron. Phys. 2, 7 (2010).

[26] W. Ebeling. Active brownian motion of pairs and swarms of particles, Acta Physica Polonica B 38, 1657 (2007).

[27] W. Ebeling, L. Schimansky-Geier. Swarm dynamics, attractors and bifurcations of active Brownian motion, Eur. Phys. J. ST 157, 17 (2008).

[28] F. Schweitzer, J.A. Holyst. Modelling collective opinion formation by means of active Brownian particles, Eur. Phys. J. B 15, 723 (2000).

[29] B. Hagen, R. Wittkowski, H. Löwen. Brownian dynamics of a self-propelled particle in shear flow, Phys.Rev. E 84, 031105 (2011).

[30] A. Glück, H. Hüffel, S. Ilijic. Canonical active Brownian motion, Phys. Rev. E 79, 021120 (2009).

[31] A. Glück, H. Hüffel, S. Ilijic. Swarms with canonical active Brownian motion, Phys. Rev. E 83, 051105 (2011).

[32] M.L. Deng, W.Q. Zhu. Stationary motion of active Brownian particles, Phys. Rev. E 69, 046105 (2004).

[33] W.Q. Zhu, M.L. Deng. Stationary swarming motion of active Brownian particles in parabolic external potential, Physica A 354, 127 (2005).

[34] K. Kreith, Oscillation Theory, Lecture Notes in Mathematics Vol. 324, (Springer-Verlag, Berlin, 1973).

[35] V.I. Arnold, Mathematical Methods of Classical Mechanics, (Springer-Verlag, Berlin, 1989).

[36] L.D. Landau, E.M. Lifshitz, Quantum Machanics. Nonrelativistic theory, (Elsevier Science Ltd., Oxford, 1991). 